\title{
Targeted Quantification of the Lysosomal Proteome in Complex Samples
}

\author{
Peter Mosen ${ }^{\dagger}$, Anne Sanner ${ }^{\dagger}$, Jasjot Singh and Dominic Winter *(D) \\ Institute for Biochemistry and Molecular Biology, Medical Faculty, University of Bonn, 53115 Bonn, Germany; \\ pmos@uni-bonn.de (P.M.); anne.sanner@uni-bonn.de (A.S.); jsin@uni-bonn.de (J.S.) \\ * Correspondence: dominic.winter@uni-bonn.de; Tel.: +49-228-73-7081 \\ + These authors contributed equally.
}

check for

updates

Citation: Mosen, P.; Sanner, A.; Singh, J.; Winter, D. Targeted Quantification of the Lysosomal Proteome in Complex Samples. Proteomes 2021, 9, 4. https://doi.org/ $10.3390 /$ proteomes 9010004

Academic Editor: Jörg Reinders

Received: 23 December 2020

Accepted: 21 January 2021

Published: 26 January 2021

Publisher's Note: MDPI stays neutral with regard to jurisdictional claims in published maps and institutional affiliations.

Copyright: (c) 2021 by the authors. Licensee MDPI, Basel, Switzerland. This article is an open access article distributed under the terms and conditions of the Creative Commons Attribution (CC BY) license (https:// creativecommons.org/licenses/by/ $4.0 /)$.

\begin{abstract}
In eukaryotic cells, lysosomes play a crucial role in the breakdown of a variety of components ranging from small molecules to complex structures, ascertaining the continuous turnover of cellular building blocks. Furthermore, they act as a regulatory hub for metabolism, being crucially involved in the regulation of major signaling pathways. Currently, 450 lysosomal proteins can be reproducibly identified in a single cell line by mass spectrometry, most of which are low-abundant, restricting their unbiased proteomic analysis to lysosome-enriched fractions. In the current study, we applied two strategies for the targeted investigation of the lysosomal proteome in complex samples: data-independent acquisition (DIA) and parallel reaction monitoring (PRM). Using a lysosomeenriched fraction, mouse embryonic fibroblast whole cell lysate, and mouse liver whole tissue lysate, we investigated the capabilities of DIA and PRM to investigate the lysosomal proteome. While both approaches identified and quantified lysosomal proteins in all sample types, and their data largely correlated, DIA identified on average more proteins, especially for lower complex samples and longer chromatographic gradients. For the highly complex tissue sample and shorter gradients, however, PRM delivered a better performance regarding both identification and quantification of lysosomal proteins. All data are available via ProteomeXchange with identifier PXDD023278.
\end{abstract}

Keywords: targeted proteomics; lysosomes; parallel reaction monitoring; data-independent acquisition; label-free quantification

\section{Introduction}

Lysosomes are membrane-bound organelles, which are well-known as the main degradative compartment of eukaryotic cells [1]. They fulfil a crucial function for the breakdown of a variety of cellular components and the recycling of their building blocks. This is achieved by $\sim 60$ hydrolases and $\sim 40$ transporters residing in the lysosomal lumen and membrane [2]. The proper function of these hydrolases is crucial for cellular homeostasis, as exemplified by the detrimental consequences of lysosomal enzyme malfunction. Mutations resulting in their altered activity, stability, or subcellular distribution can result in the accumulation of their respective substrates within lysosomes, interfering with the correct function of the organelle. Impaired lysosomal function is the primary cause of a group of $\sim 70$ inherited rare genetic diseases, so-called lysosomal storage disorders (LSDs), which frequently result in neurodegeneration, metabolic dysfunction, impaired development, and premature death [3]. To date, therapies exist only for a handful of LSDs and those available are almost exclusively symptomatic [3-5].

While the connection between lysosomal dysfunction and LSDs has been known for decades, altered lysosomal or lysosome-associated proteins have recently been shown in an increasing number of studies to be involved in more common conditions, increasing the public interest in this organelle. This includes, but is not limited to, cancer [6], neurodegenerative disorders [7], and cardiovascular diseases [8]. As part of this development, the view on lysosomes as unregulated cellular waste bags, which persisted for decades, is currently 
transitioning towards highly mobile organelles that act as a major regulatory hub of cellular metabolism. In recent years, lysosomes have been shown to vary in their properties, to be actively transported, to interact with other organelles, and to respond to various cellular and environmental stimuli with the help of an extensive network of proteins $[2,9,10]$. This involves several key players regulating cellular growth and energy metabolism, such as the mechanistic target of rapamycin complex 1 (mTORC1) or the AMP-dependent kinase (AMPK), which are activated at the lysosomal surface [11].

These emerging roles of lysosomes have led to an increasing interest in the analysis of lysosomal proteins. For the unbiased characterization of large numbers of proteins, mass spectrometry (MS)-based proteomics is currently the method of choice, as it allows for the identification, quantification, and characterization of thousands of proteins from a given sample [12]. To date, $\sim 740$ proteins have been assigned in one way or the other to lysosomes, 300 of which are either located in the lysosomal lumen, at the lysosomal surface, or directly interact with it [13].

Lysosomal proteins are typically of low abundance and therefore frequently not covered in DDA whole proteome shotgun analyses. The most common way to increase the coverage of lysosomal proteins is lysosome enrichment, resulting in a reduced sample complexity and therefore facilitating their analysis. Several lysosome enrichment methods are currently available, the most common of which are based either on density gradient centrifugation, superparamagnetic iron oxide nanoparticles (SPIONs) in combination with magnetic columns, or the immunoaffinity enrichment of tagged lysosomal proteins [14]. While all of these approaches allow for a certain degree of enrichment, they come with restrictions that limit the samples they can be applied to. While density gradient centrifugation can be performed for virtually any starting material, recovery is low and high amounts of contaminating organelles, mostly mitochondria, are included in the lysosomecontaining fractions [14]. The use of SPIONs, which are taken up by unspecific fluid phase endocytosis and delivered to the lysosomal compartment through the endocytic pathway [15], is limited to cells grown in culture which actively perform fluid phase endocytosis. Furthermore, with this approach, only those lysosomes can be isolated that receive cargo from late endosomes, which may be affected when studying models of LSDs with impaired endosome-lysosome fusion. For the immunoaffinity enrichment of lysosomes through tagged membrane proteins $[16,17]$, a fusion protein has to be stably expressed in cells or animals, requiring the generation of the respective organism. Furthermore, only lysosomes expressing the protein of choice are covered, which may result in a selection bias, and the overexpression of the tagged protein may influence lysosomal properties. For all approaches, millions of cells or milligram amounts of tissue are needed as starting material, excluding low-abundant samples from these analyses. As many LSDs affect distinct populations of cells, and the majority of LSDs can only be simulated in animal models, these limitations stall the proteomic investigation of LSDs, as it is frequently not possible to obtain lysosome-enriched fractions in sufficient quantities.

The need for enrichment of lysosomal proteins arises from the limitations of untargeted data-dependent acquisition (DDA)-based acquisition strategies, as highly abundant peptides prevent the fragmentation, and therefore identification, of those originating from low-abundant lysosomal proteins. Therefore, a promising alternative for the characterization of lysosomal proteins from small amounts of complex samples are targeted proteomics strategies. Currently, two major approaches for targeted proteomics are applied. On the one hand, previously defined peptides are fragmented in single, multiple, or parallel reaction monitoring (SRM, MRM, PRM) experiments, and abundance is determined based on the intensity of their fragment ions [18]. On the other hand, unbiased fragmentation of pre-determined $\mathrm{m} / \mathrm{z}$ windows is performed in data-independent acquisition (DIA) approaches, and the abundance of the respective peptides is determined from unique fragment ions identified in mixed MS/MS spectra [19]. In comparison to DDA-based label-free quantification strategies, PRM and DIA approaches offer increased sensitivity and reproducibility for low-abundant peptides in complex samples [20-22], making them 
ideal candidates for the analysis of lysosomal proteins from cell or tissue samples without prior enrichment.

So far, to our knowledge, targeted approaches have not been frequently used for the investigation of the lysosomal proteome. PRM was applied in a few studies for the investigation of selected lysosomal proteins (e.g., [23-25]), while only DIA approaches have been used for the analysis of the whole lysosomal proteome, so far solely investigating lysosome-enriched samples $[14,16,26]$. While it was reported that DIA is able to identify and quantify $>10,000$ proteins within a single run [27], the reproducible quantification of lysosomal proteins suffers in highly complex samples and the achievable performance in whole cell lysates is significantly lower compared to lysosome-enriched fractions [14].

In the present study, we compared DIA and PRM for the analysis of the lysosomal proteome from samples of different complexities. We investigated lysosome-enriched fractions, as well as whole cell and liver lysate, and systematically compared the performance of DIA and PRM. While we could detect lysosomal proteins with both approaches in all sample types, and DIA identified higher numbers for most samples, PRM showed a better performance in liver lysate allowing for the detection of quantitative changes which were not identified by DIA.

\section{Material and Methods}

\subsection{Cell Culture Experiments and Sample Lysis}

All cell culture experiments were performed under a sterile hood and all solutions were pre-warmed to $37^{\circ} \mathrm{C}$. Mouse embryonic fibroblasts (MEFs) were cultured at $37^{\circ} \mathrm{C}$ and $5 \% \mathrm{CO}_{2}$ in Dulbecco's modified eagle medium (DMEM), supplemented with $10 \%$ $(v / v)$ fetal calf serum (FCS), $100 \mathrm{IU} / \mathrm{mL}$ penicillin, and $100 \mu \mathrm{g} / \mathrm{mL}$ streptomycin. For the generation of MEF whole cell lysate samples, $1.5 \times 10^{6}$ cells each were seeded on three $15 \mathrm{~cm}$ plates and cultivated for $72 \mathrm{~h}$. The cells were washed once with $5 \mathrm{~mL}$ of ice-cold $1 \times$ phosphate-buffered saline (PBS), scraped in $600 \mu \mathrm{L}$ of ice-cold PBS, and collected in a $1.5 \mathrm{~mL}$ microtube. Cells were pelleted by centrifugation at $1000 \times \mathrm{g}$ and $4{ }^{\circ} \mathrm{C}$ for $4 \mathrm{~min}$, the supernatant was discarded, and the cell pellet was re-suspended in $600 \mu \mathrm{L}$ lysis buffer (4\% SDS, $100 \mathrm{mM}$ HEPES pH 7.5). Subsequently, the cell suspension was incubated at $95^{\circ} \mathrm{C}$ for $10 \mathrm{~min}$ followed by sonication using a Ultrasonics Sonifier 250 (Branson, Danbury, CT, USA) at a duty cycle of $60 \%$ and an output of 6 for $90 \mathrm{~s}$. Samples were centrifuged at $20,000 \times g$ and RT for $30 \mathrm{~min}$ and the clear supernatants were transferred to new microtubes.

Lysosome isolation was performed from MEF cells using SPIONs as described elsewhere [26]. In brief, cells were cultivated in DMEM with $2.5 \%$ FCS for $72 \mathrm{~h}\left(3 \times 10^{6}\right.$ cells per $10 \mathrm{~cm}$ dish), $1 \mathrm{~mL}$ of magnetite solution (EndoMAG40, Liquids Research, North Wales, UK) was added to each plate, and the cells were incubated for $24 \mathrm{~h}$ (pulse period). Subsequently, the cells were washed twice with pre-warmed PBS, fresh DMEM (10\% FCS) was added, and the cells were incubated for $24 \mathrm{~h}$ (chase period). Cells were washed with ice-cold PBS and harvested using a cell scraper in $2 \mathrm{~mL}$ lysosome isolation buffer $(250 \mathrm{mM}$ sucrose, $10 \mathrm{mM}$ HEPES pH 7.4, $1 \mathrm{mM} \mathrm{CaCl} 2,15 \mathrm{mM} \mathrm{KCl}, 1 \mathrm{mM} \mathrm{MgCl} 2,1.5 \mathrm{mM} \mathrm{MgAc}, 1 \mathrm{mM}$ dithiothreitol (DTT), 1x cOmplete EDTA-free protease inhibitor cocktail (Roche Diagnostics, Mannheim, Germany)). Plasma membranes were disrupted using a dounce homogenizer, and lysosomes were enriched using Miltenyi LS columns (Miltenyi Biotech, Auburn, CA) and eluted using a plunger.

\subsection{Preparation of Mouse Liver Samples}

Mice were handled in accordance with local regulations concerning the welfare of animals. Three months-old male C57BL/6 mice were sacrificed by cervical dislocation, the liver was extracted, and snap-frozen in liquid nitrogen. The frozen tissue was chopped into small pieces using a razor blade, and $1 \mathrm{~mL}$ of lysis buffer (4\% SDS, $100 \mathrm{mM}$ HEPES $\mathrm{pH} 7.5$ ) was added. The sample was incubated for $10 \mathrm{~min}$ at $95^{\circ} \mathrm{C}$ and sonicated using an Ultrasonics Sonifier at a duty cycle of $60 \%$ and an output of 6 for 90 s. Subsequently, 
the samples were again incubated at $95^{\circ} \mathrm{C}$ for $10 \mathrm{~min}$, centrifuged at $20,000 \times g$ and RT for $30 \mathrm{~min}$, and the clear supernatants were transferred to new microtubes.

\subsection{Sample Preparation for Mass Spectrometry}

The protein concentration of all samples was determined using the DC Protein Assay (Bio-Rad Laboratories, CA, USA). For MEF whole cell lysate and liver samples, $100 \mu \mathrm{g}$ of protein were used for each replicate while $20 \mu \mathrm{g}$ were used for lysosome-enriched fractions. Sample volumes were adjusted to $200 \mu \mathrm{L}$ using HPLC-grade water and proteins were precipitated by addition of $1 \mathrm{~mL}$ ice-cold chloroform/methanol (2:1 v/v), vigorous vortexing, and centrifugation at $20,000 \times g, 4{ }^{\circ} \mathrm{C}$ for $1 \mathrm{~h}$. The liquid phases were discarded, the protein pellets washed once with $1 \mathrm{~mL}$ of ice-cold methanol, and centrifuged at 20,000 $\times g$, $4{ }^{\circ} \mathrm{C}$ for $15 \mathrm{~min}$, followed by the removal of methanol. Protein pellets were air-dried and solubilized in 1\% RapiGest (Waters, Milford, MA, USA), $0.1 \mathrm{M} \mathrm{NH}_{4} \mathrm{HCO}_{3} \mathrm{pH} 7.8$ at $95{ }^{\circ} \mathrm{C}$ for $10 \mathrm{~min}$. Subsequently, samples were diluted 1 to 5 with $0.1 \mathrm{M} \mathrm{NH}_{4} \mathrm{HCO}_{3}$ and trypsin (Promega, Mannheim, Germany) was added at an enzyme-to-substrate ratio of 1:500, followed by incubation at $37^{\circ} \mathrm{C}, 800 \mathrm{rpm}$ in a thermomixer for $45 \mathrm{~min}$. Proteins were reduced using DTT ( $5 \mathrm{mM}$ final concentration) at $56^{\circ} \mathrm{C}$ for $30 \mathrm{~min}$ and alkylated with acrylamide (20 mM final concentration) for $30 \mathrm{~min}$ at RT, followed by quenching of the reaction through the addition of $5 \mathrm{mM}$ DTT. Finally, trypsin was added at an enzyme-to-sample ratio of 1:50 and the RapiGest concentration adjusted to $0.1 \%$ using $0.1 \mathrm{M} \mathrm{NH}_{4} \mathrm{HCO}_{3}$. Proteins were digested overnight at $37^{\circ} \mathrm{C}$, and on the following day, RapiGest was hydrolyzed by addition of $1 \%$ TFA (final concentration) and incubation in a thermomixer at $800 \mathrm{rpm}$, $37^{\circ} \mathrm{C}$ for $30 \mathrm{~min}$. Hydrolyzed RapiGest was precipitated by centrifugation at $20,000 \times g$, RT for 10 min and the supernatants were desalted using Oasis HLB cartridges (Waters) as described elsewhere [28]. Briefly, cartridges were equilibrated with $70 \% \mathrm{ACN}, 0.1 \%$ acetic acid (AA), washed with $0.1 \%$ AA, and the sample was loaded. Subsequently, cartridges were washed with $0.1 \%$ AA and peptides were eluted sequentially with $30 \%, 50 \%$, and $70 \% \mathrm{ACN}, 0.1 \%$ AA. Eluate fractions were pooled and the combined samples dried in a vacuum centrifuge. Dried peptides were re-suspended in $5 \% \mathrm{ACN}$, and the peptide concentration was determined using the Quantitative Fluorometric Peptide Assay (ThermoFisher Scientific, Waltham, MA, USA), and the peptides were dried again.

\subsection{LC-MS/MS Analysis}

All analyses were performed using a Dionex Ultimate 3000 nano-UHPLC system coupled to an Orbitrap Fusion Lumos mass spectrometer (both Thermo Fisher Scientific). Analytical columns were produced in-house as follows: spray tips were generated with a P-2000 laser puller (Sutter Instruments, Novato, CA) from $360 \mu \mathrm{m}$ outer diameter and $100 \mu \mathrm{m}$ inner diameter fused silica capillaries and packed to a length of $40 \mathrm{~cm}$ with $3 \mu \mathrm{m}$ ReprosilPur AQ $C_{18}$ particles (Dr. Maisch, Ammerbuch-Entringen, Germany). Dried peptides were reconstituted in 5\% ACN, 5\% formic acid (FA), and $1 \mu \mathrm{g}$ was loaded together with $750 \mathrm{fmol}$ of internal retention time standards (iRTs, Biognosys, Schlieren, Switzerland) to the analytical column at a flow rate of $600 \mathrm{nl} / \mathrm{min}$ with $100 \%$ solvent $\mathrm{A}(0.1 \%$ FA in water $)$ for 25 min. Peptides were eluted with 60, 120, and 240 min linear gradients from 5-35\% solvent $\mathrm{B}(90 \% \mathrm{ACN}, 0.1 \% \mathrm{FA})$ at a flow rate of $300 \mathrm{nl} / \mathrm{min}$. For parallel reaction monitoring (PRM) measurements, precursor masses were selected from a previously recorded dataset (Table S1, [26]) while the spectral library for data-independent acquisition (DIA) analyses was generated using 240 min data-dependent acquisition (DDA) runs. In these analyses, survey spectra were acquired with a mass range of m/z 350-1200 at a resolution of 60,000 and an AGC target setting of $4 \times 10^{5}$ The most abundant precursor ions (charge states of 2-4) were isolated using the quadrupole (isolation width of $\mathrm{m} / \mathrm{z} 1.6$ ), and fragmented by HCD with a collision energy of 27 in the top speed mode (cycle time of $3 \mathrm{sec}$ ). Fragment ion spectra were acquired in the Orbitrap mass analyzer at a resolution of 30,000 and fragmented ions were excluded from further fragmentation for $120 \mathrm{~s}$. For DIA analyses, one MS scan was performed with a mass range of $\mathrm{m} / \mathrm{z} 350-1200$, a resolution of 120,000 , 
a maximum injection time of $20 \mathrm{~ms}$, and an AGC target setting of $5 \times 10^{5}$. The MS scan was followed by static DIA MS/MS scans, covering the same $\mathrm{m} / \mathrm{z}$ range with an overlap of $\mathrm{m} / \mathrm{z} 0.5$, with the following gradient lengths/ scan numbers/ isolation windows/ cycle times: $60 \mathrm{~min} / 24 \mathrm{scans} / \mathrm{m} / \mathrm{z} 35.9 / 2.34 \mathrm{~s} ; 120 \mathrm{~min} / 36 \mathrm{scans} / \mathrm{m} / \mathrm{z} 24.1 / 3.44 \mathrm{~s}$; $240 \mathrm{~min} / 58 \mathrm{scans} / \mathrm{m} / \mathrm{z} 15.2 / 5.45 \mathrm{~s}$ ). The HCD collision energy was set to $27 \%$ and DIA MS/MS scans were acquired in the Orbitrap with a resolution of 30,000, a maximum injection time of $60 \mathrm{~ms}$, and an AGC target setting of $1 \times 10^{6}$. For PRM analyses, MS spectra were acquired with a mass range of $\mathrm{m} / \mathrm{z} 300-1500$ at a resolution of 60,000, a maximum injection time of $118 \mathrm{~ms}$, and an AGC target setting of $4 \times 10^{5}$. Peptides were isolated in the quadrupole with an isolation width of $\mathrm{m} / \mathrm{z} 1.2$ and fragmented by HCD with a collision energy of $27 \%$. MS $/$ MS scans were acquired in the Orbitrap mass analyzer with a mass range of $\mathrm{m} / \mathrm{z} 200-2000$, a resolution of 30,000, a maximum injection time of $54 \mathrm{~ms}$ and an AGC target setting of $5 \times 10^{4}$.

\subsection{Data Analysis}

For DIA library generation, DDA *.raw files were analyzed with the Pulsar search engine integrated in Spectronaut (Version: 14.7.20, Biognosys) (1). Uniprot Mus musculus (release date: 09.09.2019 with 17,023 entries), in combination with a database containing common contaminants, was used for database searching with Spectronaut standard settings [29]. In brief, cleavage by trypsin with up to two missed cleavage sites was defined, propionamide (cysteine) was set as fixed and oxidation (methionine) as variable modification, and three to six fragment ions were selected for library generation, dependent on the intensity of the respective peptide. The high-precision iRT concept (dynamic) was applied for retention time alignment. Matching of mass tolerances for precursors, fragment ions, as well as peak extraction windows were determined automatically by Spectronaut. Only MS precursor information was utilized for peak detection, and interference correction was enabled. Global normalization was performed for individual runs based on the median abundance. Data were filtered with a $1 \%$ FDR cut off on the precursor and protein level (q-value < 0.01) [30]. $p$-value determination and unsupervised clustering were performed with the post-analysis pipeline of Spectronaut applying default parameters (distance metric: Manhattan Distance; linkage strategy: Ward's method; multiple testing correction: Storey's method).

For PRM analyses, a spectral library was generated using a subset of our previously published DDA dataset [26] with Skyline [31], applying a cut-off score of 0.95. Ambiguous peptide matches were excluded, and the library was filtered for peptides which were previously manually selected to be included in the assay (Table S1). For analysis of PRM data, ${ }^{*}$.raw files were loaded into Skyline daily version 20.2.1.315. Automated fragment ion selection by Skyline was utilized (6 ions/peptide) with the exception of the peptides with the sequence SLQPLYR and GSFSLSVR, for which only 5 fragment ions matched, using the following criteria: maximum mass error of $10 \mathrm{ppm}$ for MS and MS/MS ion trace filtering (centroid mode) and charge states of $1+/ 2+$ for $b$ - and y-ions as well as $2+/ 3+$ for precursor ions. Integration boundaries of iRT peptides were inspected manually and corrected, if necessary. Experimental data were only reviewed when Skyline reported a peak truncation, and peptides with truncated peaks or no MS/MS signal were excluded from further analysis. Peptide-centric reports were exported and further processed in MS Excel. For peptide and protein quantification, the summed area under the curve (AUC) of fragment ions was used. For all analyses, only peptides with quantitative values in all three replicates were considered.

\section{Results and Discussion}

We showed previously that the analysis of lysosome-enriched fractions with DIA allows for a superior performance compared to DDA measurements in a reduced amount of time [26]. When we investigated the lysosomal proteome in samples of higher complexity (such as whole cell lysates); however, we observed that the number of lysosomal 
proteins that can be reproducibly identified and quantified was markedly lower, indicating a reduced performance in such samples [14]. This is most likely due to the fact that co-fragmenting peptides increase the complexity of MS/MS spectra, which results in a decreased performance for the quantification of lysosomal proteins, as they are of low abundance relative to the whole proteome. In theory, PRM approaches should be superior in this aspect, as only a small $\mathrm{m} / \mathrm{z}$ window, that is specific for the individual peptide, is fragmented.

In order to determine which strategy is best-suited for the MS/MS-based quantification of the lysosomal proteome in samples with different complexities, we compared DIAand PRM-based quantification (Figure 1). Initially, we defined a highly reproducible lysosomal proteome from a dataset generated previously by our group, comprising 39 DDA LC-MS/MS runs of lysosome-enriched fractions from MEFs [26]. From these data, we only considered proteins which were assigned to the lysosomal compartment based on gene ontology (GO) and Uniprot categories, and which were detected in $\geq 75 \%$ of LCMS/MS runs with $\geq 2$ unique peptides, resulting in a final list of 374 proteins (Table S1). For the comparison of DIA and PRM, we used a lysosome-enriched fraction from MEFs (LEF) as benchmark samples, as it contains the highest percentage of lysosomal proteins. Furthermore, we used MEF whole cell lysate (MWCL) as well as liver tissue lysate (LTL), representing samples of increasing complexity. We performed all experiments in triplicates with independent experimental replicates for each measurement.

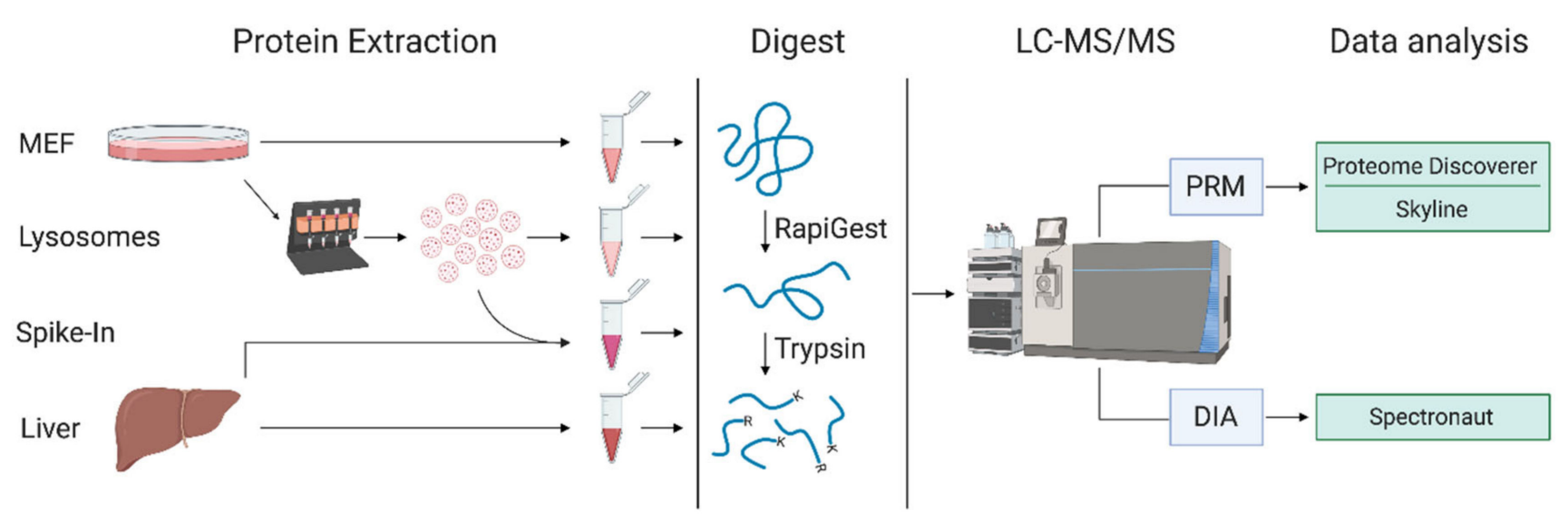

Figure 1. Workflow for sample preparation and analysis. For each sample type, proteins were extracted and digested in three experimental replicates and analyzed by LC-MS/MS using either DIA or PRM with different gradient lengths. MEF: mouse embryonic fibroblasts; DIA: data independent acquisition; PRM: parallel reaction monitoring; K: lysine; R: arginine. Created with Biorender.com.

\subsection{Gradient Length and Sample Complexity Affect Lysosomal Protein Quantification by DIA}

Initially, we analyzed all three sample types with different gradient lengths $(60 \mathrm{~min}$, $120 \mathrm{~min}$, and $240 \mathrm{~min}$ ) by DIA. We adjusted the width of DIA fragmentation windows depending on the gradient length in order to allow for a similar number of data points across chromatographic peaks of the individual gradients. Consequently, a shorter gradient resulted in a larger $\mathrm{m} / \mathrm{z}$ window and vice versa, influencing the number of co-fragmented precursor ions. To assess performance of the individual methods, we evaluated both the numbers of total proteins, and those previously reported to be lysosomal (Table S1) that were identified in each run (Table S2).

We found highest total protein numbers in the MWCL, followed by the LEF, and the LTL (Figure 2a). While we observed a steady increase in the number of identified total proteins from $60 \mathrm{~min}$ to both $120 \mathrm{~min}$ and $240 \mathrm{~min}$ gradients for MWCL (increase of $19 \%$ and $28 \%$ ) and LTL (increase of $28 \%$ and $46 \%$ ) samples, the numbers of IDs detected in the LEF only increased from $60 \mathrm{~min}$ to $120 \mathrm{~min}$ gradients (increase of $30 \%$ ). When 
considering only lysosomal proteins, we identified, as expected, highest numbers in the LEF, followed by MWCL and LTL. While the latter two showed a similar correlation of gradient length and protein identifications, the LEF produced virtually constant numbers for all gradients and only CV values improved. The differences in identifications were particularly pronounced when considering lysosomal proteins quantified with $<5 \% \mathrm{CV}$ in the 60 min gradient analyses, 116 of which were found in the LEF but only 45 in the LTL (Figure 2b).
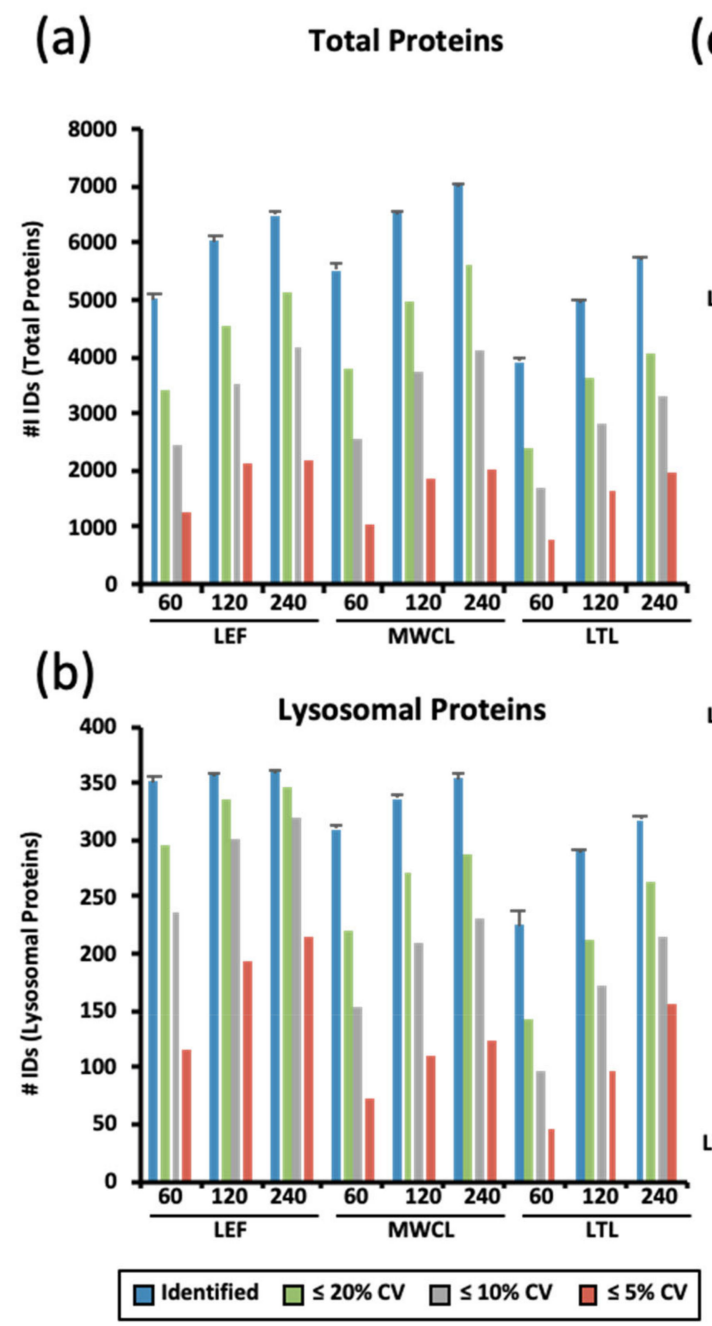

(c) Total Proteins
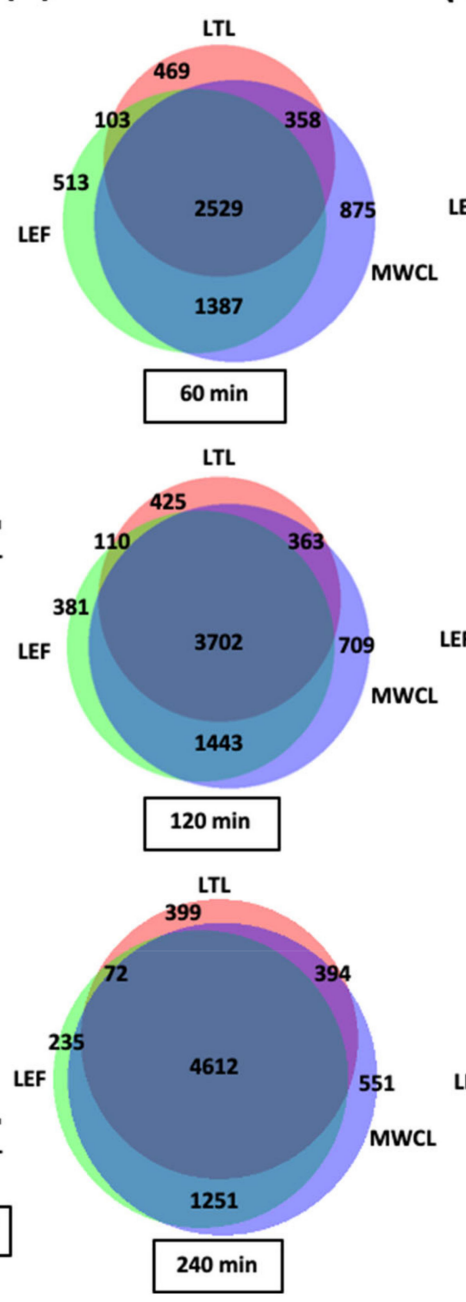

(d) Lysosomal Proteins

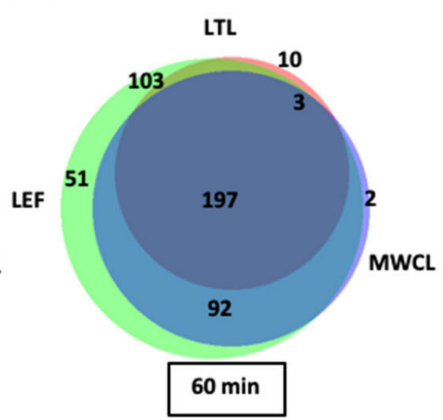

LTL

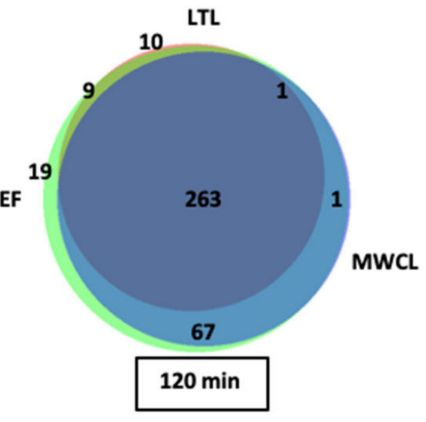

LTL

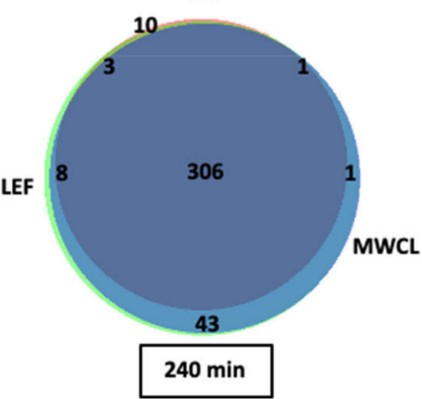

Figure 2. Identification of total and lysosomal proteins in DIA experiments. (a,b) Numbers of identified total proteins (a) and those known to be located at/in the lysosome $(\mathbf{b})$. Shown are average values $(n=3)$ for the analysis of lysosome-enriched fractions of MEF (LEF), MEF whole cell lysate (MWCL), and liver tissue lysate (LTL) with three different gradient lengths. Total IDs as well as those quantified with a CVs $\leq 20 \%, 10 \%$, and $5 \%$ are shown. (c,d) Overlap in protein identification for total proteins (c) and those known to be located at the lysosome (d) for proteins identified in all three replicates of LEF, MWCL, and LTL samples analyzed with different gradient lengths. Venn diagrams were generated with the tool BioVenn [32]. LEF: lysosome-enriched fractions from mouse embryonic fibroblasts; MWCL: mouse embryonic fibroblast whole cell lysates; LTL: whole liver tissue lysate; CV: coefficient of variation; ID: number of identified proteins.

It was quite surprising to us that we identified the highest number of proteins in the MWCL, as the LTL should theoretically be the most complex sample. A possible explanation for this observation is that the LTL contains a certain number of highly abundant proteins, which account for a larger percentage of the sample than highly abundant MWCL and LEF proteins. Consequently, in LTL the remaining proteins present a smaller fraction of the total 
sample. As the C-trap - which is used for ion storage prior to injection into the Orbitrap has a limited capacity [33], this results in reduced fragment ion intensities for the lower abundant proteins, which are not sufficient for identification/quantification. Furthermore, the highly abundant fragment ions from these proteins dominate the DIA MS/MS spectra, resulting in reduced detection of co-fragmented lower abundant peptides.

When considering the increase in identification of total unique proteins with increasing analysis time, each sample contributed a distinct population (Figure 2c). Lysosomal protein identifications, on the other hand, were very similar between the samples, and for $240 \mathrm{~min}$ gradients the majority was identified at least in both the MWCL and the LEF (Figure 2d). These results imply that short gradients suffice to achieve a good coverage of lysosomal proteins in LEFs, while longer gradients are needed when MWCL and LTL are analyzed.

While this confirms that lysosomal proteins are more abundant in lysosome-enriched fractions, which was certainly expected, it also shows that the detection of such lower abundant proteins in DIA analyses suffers from sample complexity. As our DIA analyses were performed with different $\mathrm{m} / \mathrm{z}$ windows for the different gradient lengths, this effect is most likely related to the number of co-fragmented precursor ions and the resulting MS/MS spectrum complexity. The bigger the fragmentation window is, the more peptides are co-fragmented, and consequently the fragment ions of the lower abundant lysosomal proteins are identified with a lower efficiency.

\subsection{Variation of Protein Abundance and Variance between Sample Types in DIA Analyses}

To further follow up on this effect, we investigated the protein abundances for the individual samples utilizing median-normalized AUCs (Figure 3a). Confirming our previous assumption, the liver lysate resulted in the highest average abundance (1.6- and 1.8-fold higher compared to the lysosome-enriched fractions and MEF lysate, respectively, for 60 min gradients) and the largest number of highly abundant proteins (36 proteins compared to 11 and 12 with $\log 10$ values $>7.5$ for LEF and MWCL, respectively). Average protein abundance correlated inversely with the number of protein identifications, with highest values in the shortest gradient, irrespective of the sample type. For lysosomal proteins, we observed highest average abundances in the LEF (1.8- and 2.4-fold higher compared LTL and MWCL for 60 min gradients) and, unlike the total protein identifications, no decrease in abundance with increasing gradient length (Figure $3 \mathrm{c}$ ). Average CV values, however, behaved similarly for all types of proteins (Figure 3b,d).

To visualize the differences of the individual datasets on a global scale, we generated heatmaps for the average abundances of total and lysosomal proteins, clustered in a rowand column-wise manner (Figure 4a,b). For both analyses, we observed distinct protein populations which formed individual clusters, based on their abundance in the respective sample types and gradient lengths. In most cases, gradient length played a decisive role, while the highest differences existed for the LTL relative to the other samples. For the majority of known lysosomal proteins, we detected a higher abundance in the LEF relative to the MWCL and the LTL, while certain proteins were exclusively identified in the LEF. We also identified, however, some clusters with a higher abundance in MWCL and LTL, implying that either not all lysosomal proteins were recovered efficiently in the lysosomeenrichment step, or that a certain population of these proteins was located in a different cellular compartment. 

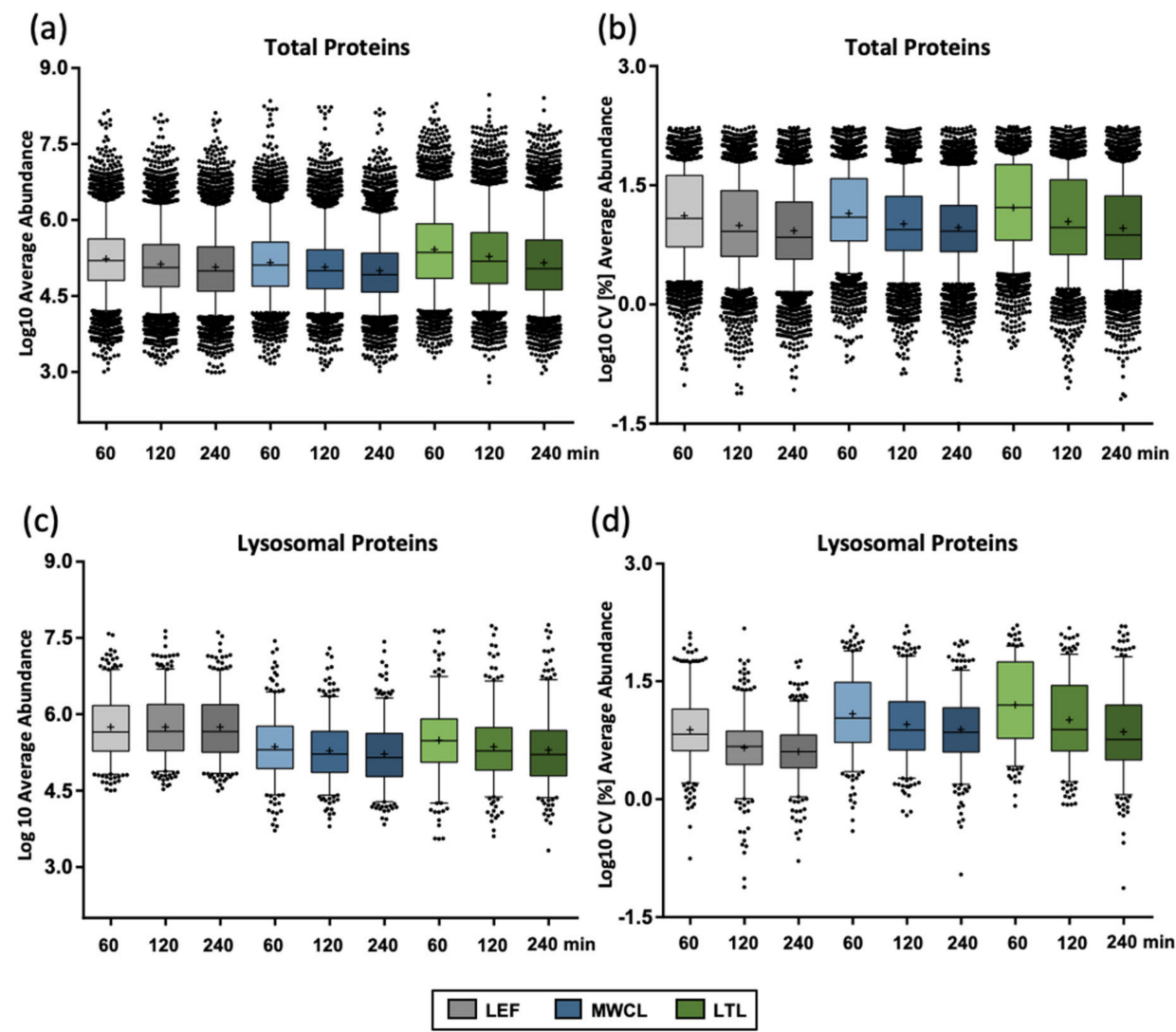

Figure 3. Reproducibility of protein abundance in DIA analyses. (a) Protein abundance for total proteins identified in individual samples with different gradient lengths. (b) CV values for total proteins identified in individual samples with different gradient lengths. (c) Protein abundance for lysosomal proteins identified in individual samples with different gradient lengths. (d) CV values for lysosomal proteins identified in individual samples with different gradient lengths. Shown are combined values from 3 replicates, the median is indicated by a line, while the average is marked with a "+". LEF: lysosome-enriched fractions from mouse embryonic fibroblasts; MWCL: mouse embryonic fibroblast whole cell lysates; LTL: whole liver tissue lysate; CV: coefficient of variation.

(a)

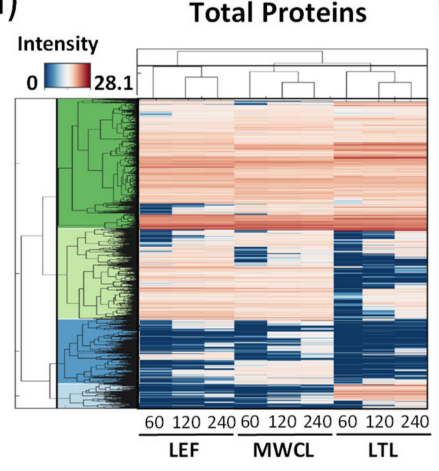

(b)

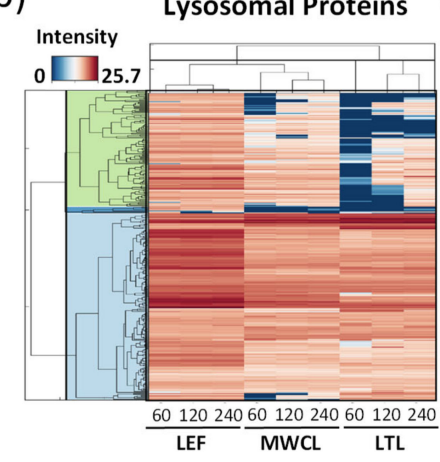

(c)

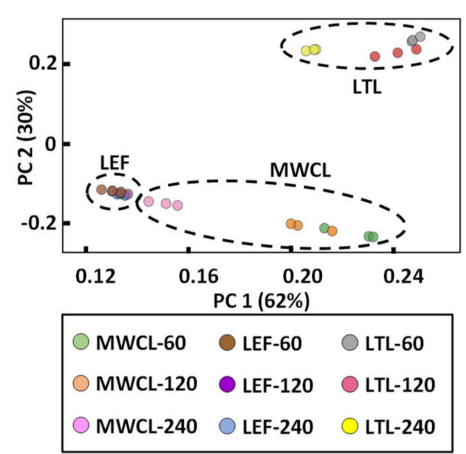

Figure 4. Global analysis of DIA datasets. (a,b) Unsupervised clustering of average abundances (columns) of LEF, MWCL, and LTL for three different gradient lengths (60,120 and $240 \mathrm{~min})$ for total proteins identified $((\mathbf{a}), \mathrm{n}=7145)$ and lysosomal proteins $((\mathbf{b}), \mathrm{n}=314)$. The color code indicates the normalized intensity of the individual proteins. (c) Principal component analysis (PCA) for all analyses with two defined standardized principal components (PC1 and PC2). LEF: lysosome-enriched fractions from mouse embryonic fibroblasts; MWCL: mouse embryonic fibroblast whole cell lysates; LTL: whole liver tissue lysate. 
Finally, we assessed the global variability between the datasets by principal component analysis (PCA, Figure 4c). The two main principal components (PC1 and PC2), which are responsible for $62 \%$ and $30 \%$ of the variance in the dataset, allowed for a good separation of the samples. As individual replicates of the same sample type and gradient length clustered closely together, the main variance in the dataset (PC1) can be explained based on the difference of the sample type itself. However, especially for the MWCL, the $240 \mathrm{~min}$ gradient data behaved significantly differently than those acquired with other gradients, being actually closer to the LEF. This relates most likely to the fact that the LEF originated from MEFs and that proteome coverage in the $240 \mathrm{~min}$ gradient increased to such an extent that very similar proteins were identified (Figure 2c).

\subsection{PRM Assay Development}

For the 374 proteins included in our lysosomal proteome reference list (Table S1), 10,141 unique peptides were identified in the course of our previous analysis [26]. We narrowed down the list of putative peptides by excluding those identified with variable modifications, missed cleavage sites, or containing the amino acid combination PK or PR (as proline residues interfere with tryptic cleavage). These criteria were fulfilled by 3816 peptides representing 367 proteins. Based on the average signal intensities in this dataset, we considered the two most abundant peptides for each protein, resulting in a final list of 680 peptides, as for some proteins only a single peptide fulfilled our criteria (Figure 5a, Table S1).

(a)

(b)

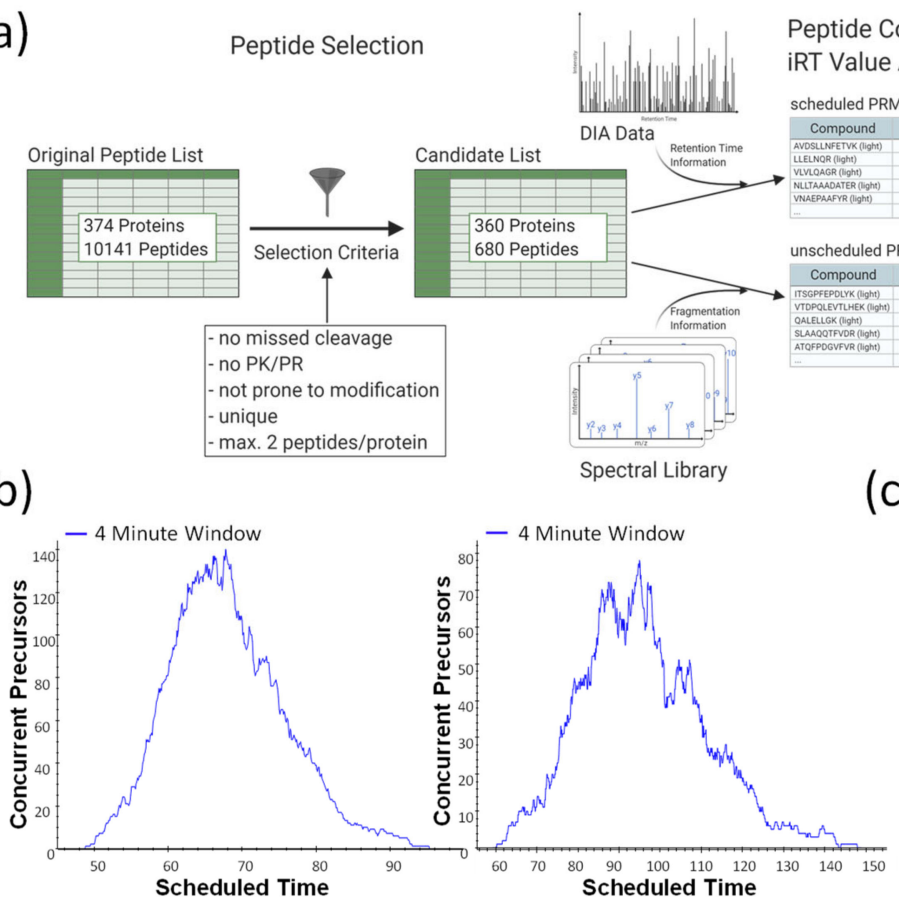

(c)

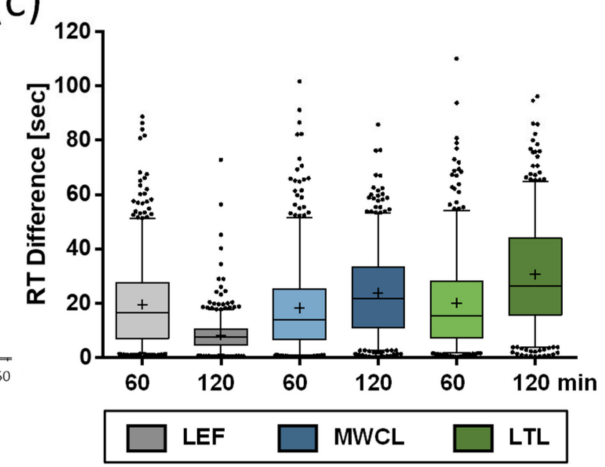

Figure 5. Establishment of PRM assay. (a) Workflow for the development of the PRM assay. Created with Biorender.com. (b) Distribution of concurrent precursor elution in $60 \mathrm{~min}$ and 120 min gradients. (c) Differences between predicted and experimentally determined retention times for individual measurements. Shown are combined values of three replicates and the mean (+), median (line), and interquartile range are indicated. LEF: lysosome-enriched fractions from mouse embryonic fibroblasts; MWCL: mouse embryonic fibroblast whole cell lysates; LTL: whole liver tissue lysate; CV: coefficient of variation; RT: retention time.

For PRM assay scheduling, we extracted the peptides' retention time information from the 120 min DIA runs of the LEF, followed by the refinement of the assay by PRM analysis of the same sample, including high-precision indexed retention time (iRT) standards [29]. 
After analysis with an initial scheduled PRM assay (15 min retention time windows), we performed unscheduled PRM runs for those peptides that were not detected in these initial analyses. Finally, we combined the acquired retention times of all peptides that we were able to detect with distinct fragment ion signatures, and created an iRT-normalized library. This resulted in a final assay comprising 586 peptides from 340 lysosomal proteins. For the analysis of acquired PRM data with Skyline [31], we built a reference spectral library from our previously measured DDA dataset of the LEF [26]. Finally, we generated two assays utilizing $4 \mathrm{~min}$ retention time windows. In one assay, all peptides were analyzed in a single 120 min gradient, while the other assay consisted of two 60 min gradients. This was necessary, as the high number of concurrent precursors (up to 140) would have drastically reduced the number of data points for chromatographic peaks eluting in the middle of gradient (Figure $5 b$ ). For subsequent analyses, we determined iRT correction factors for the different sample types using DDA runs, and adjusted the scheduling accordingly. For data export from Skyline, a minimum number of six data points was defined.

\subsection{Gradient Length and Sample Type Affect Data Quality in PRM Analyses}

Due to the high number of peptides, we defined parameters for the acceptance of PRM quantification data without manual inspection of each peptide. Initially, we assessed the difference between predicted and experimentally observed retention times. For the $60 \mathrm{~min}$ and 120 min gradients, we observed average peak widths of $21 \mathrm{sec}$ and $25 \mathrm{sec}$, respectively, and an average retention time variability of $\pm 15 \mathrm{sec}$, with a slightly lower average shift for the 60 min gradients (Figure 5c). LEF analyses with 120 min gradients presented with only \pm 8 sec an exception, which might be due to the fact that we performed the PRM assay retention time normalization with 120 min gradient measurements of the LEF, while the scheduling for MWCL and LTL was solely based on iRT predictions.

Next, we investigated the quality of acquired fragment ions for the individual peptides utilizing the dot product $(\operatorname{dotP})$ value [34], which allows for correlation between the acquired spectrum and the spectral library (generated from our reference dataset [26]). Especially for the analysis of unfractionated highly complex samples, this allows to identify the impact of interfering ions that may result in false quantification results. Across all analyses, the average dotP value was $>0.85$, indicating a good matching of our PRM data with the spectral library (Table S3). While we observed roughly similar dotP values for both the $60 \mathrm{~min}$ and $120 \mathrm{~min}$ assays, they decreased with sample complexity (Figure 6a). Compared to the LEF, which displayed the least variation, especially the LTL resulted in lower dotP values and higher variability. These findings imply a lower relative abundance of lysosomal proteins and an increase of interfering fragment ions in the MS/MS spectra for the more complex samples, which is also in agreement with the DIA data.

Subsequently, we investigated the correlation of dotP values and numbers of fragment ions used for quantification of the different sample types and gradient lengths (Figure S1). Utilizing three to six fragment ions, we applied different dotP value thresholds and determined the number of peptides passing it. As expected, lower numbers of fragment ions resulted in more peptides passing the threshold at higher dotP values. This was especially true when dotP thresholds $\geq 0.9$ were applied, as we observed a clear difference between the peptides identified with 3, 4, 5, or 6 fragment ions. For lower $\operatorname{dot} P$ values (0.7-0.8), this effect was far less pronounced. As already indicated by the average dotP values (Figure 6a), an inverse correlation with sample complexity could be observed. Based on these analyses, we defined 6 fragment ions per peptide with a dotP value of 0.7 as cut-off for the acceptance of quantification information from PRM data.

For LEF data, this cut-off resulted in an acceptance rate of $92 \%$ of the peptides included in our assay for both gradients. The value for MWCL was $87 \%$ for both gradients and for LTL 73\% and 78\%, for the $60 \mathrm{~min}$ and 120 min gradient, respectively. Applying these cut-offs, we exported the data from Skyline and utilized them for all further analyses (Table S4). 

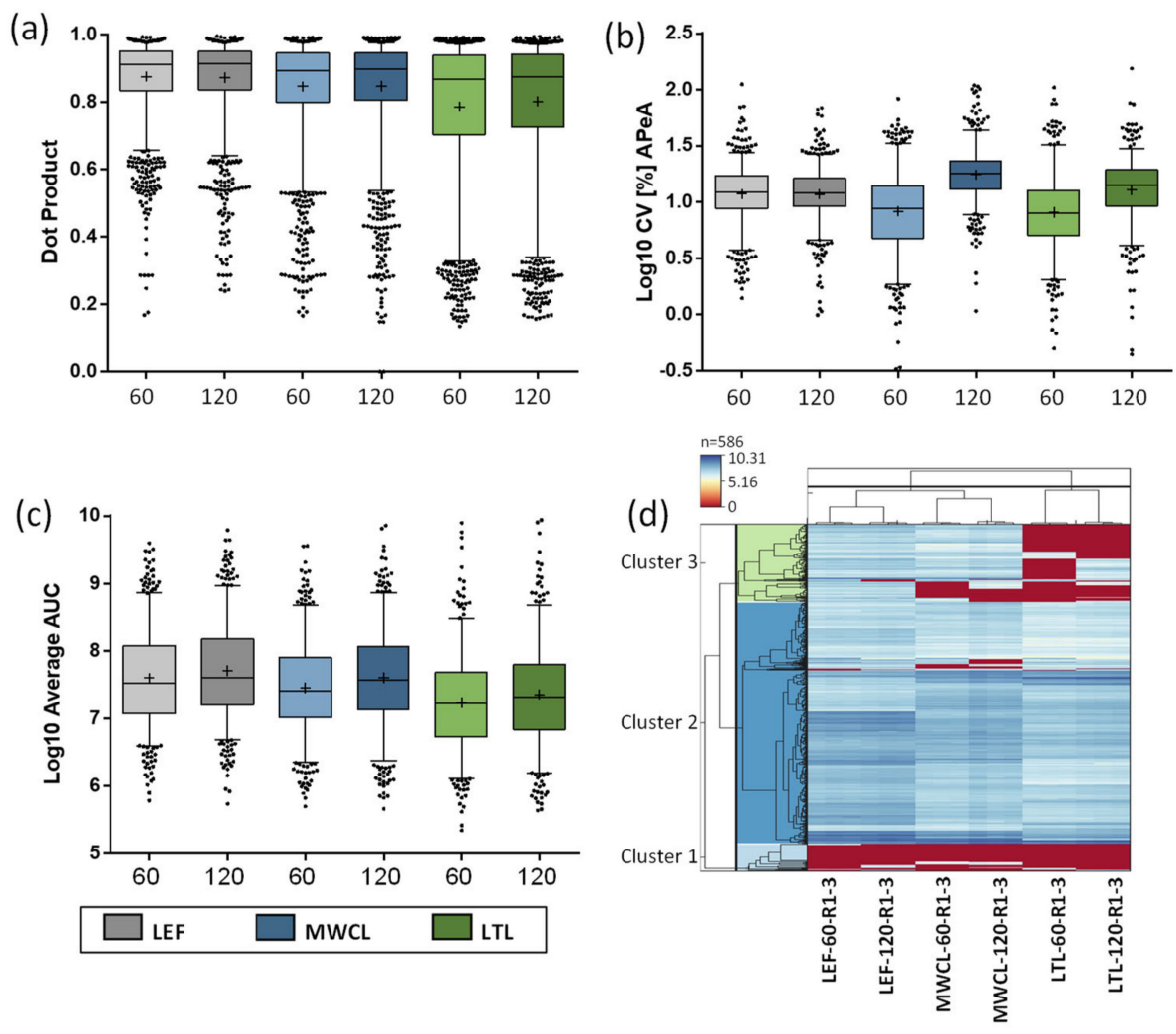

Figure 6. Characterization of data generated by PRM analysis. (a) Assessment of data quality in PRM analyses by Dot Product values. (b) Log10 CV values for the summed AUC of all targeted peptides. (c) Averaged $\log 10$-transformed summed peptide AUCs of the replicates $(n=3)$ across the different sample types and gradient lengths from PRM measurements. ( $\mathrm{a} / \mathrm{b} / \mathrm{c})$ Indicated are mean (+), median (line), and interquartile range. (d) Heatmap of the log10-transformed AUCs of all peptides covered by the PRM assay across all measurements. Each column contains data from one measurement and each row represents one peptide. Peptides are clustered if they exhibit similar trends across the samples. LEF: lysosome-enriched fractions from mouse embryonic fibroblasts; MWCL: mouse embryonic fibroblast whole cell lysates; LTL: whole liver tissue lysate; AUC: area under the curve; CV: coefficient of variation; APeA: average peptide abundance.

\subsection{PRM Analysis of the Lysosomal Proteome}

We initially investigated the reproducibility of quantification (Figure 6b). While CVs of the LEF analyses were similar for both gradients, the 120 min gradient resulted in consistently higher CVs for both MWCL and LTL. Surprisingly, the higher complexity samples resulted in a lower average CV than the LEF for the $60 \mathrm{~min}$ runs. A possible explanation for this observation is the lower sample amount utilized for LEF sample preparation $(\sim 20 \mu \mathrm{g})$ compared to MWCL and LTL $(\sim 100 \mu \mathrm{g})$, which may have resulted in a higher variability during pipetting and desalting. Subsequently, we calculated the average summed area under the curve (AUC) for each sample type and gradient length (Figure 6c). Interestingly, while we saw a higher summed abundance for the LEF in comparison to the other samples for 60 min gradients, the values were more similar for the $120 \mathrm{~min}$ analyses, especially for the comparison of LEF and MWCL. This could be related to the different numbers of data points acquired over the chromatographic peak as well as variances in peak width/shape between gradients.

Finally, we assessed the overall correlation of the data in a heatmap, depicting the signal intensities of individual peptides in each sample and replicate, clustered in a rowand column-wise manner (Figure $6 \mathrm{~d}$ ). In general, the lysosomal peptides formed three distinct clusters. Two clusters showed similar expression levels (general high or low expression) in all samples, while the third cluster contained proteins that were detected 
with differing levels in the individual samples. In agreement with the DIA data, we observed subsets of peptides that were only found in the LEF, and were not detected in both the MWCL and LTL. Moreover, peptides existed that were detected both in LEF and MWCL, but not in LTL, and a small subset with higher abundance in LTL compared to the other samples.

\subsection{Comparison of DIA- and PRM-Based Quantification of the Lysosomal Proteome}

In order to correlate the performance of DIA- and PRM-based quantification of the lysosomal proteome, we initially compared the data obtained from the individual datasets. When considering the average abundance and CVs of lysosomal proteins, we observed for all DIA analyses that longer gradients resulted in lower CV values but also lower abundances (Figure 3c,d), while for PRM both intensities and CVs (with the exception of the LEF data) increased with gradient length (Figure $6 b, c$ ). When considering how many lysosomal proteins were found in the individual analyses, we identified higher numbers for DIA, with the exception of LTL analyzed with 60 min gradients (Figure S2). To assess to what extend the abundances acquired with the individual approaches correlate, we extracted the AUCs of the peptides included in our PRM assay from the DIA dataset (60 min gradients for both approaches) and performed a direct comparison (Figure 7a). We observed for all three sample types that PRM resulted in higher signal intensities than DIA and that correlation of signal intensities was dependent on the abundance of the respective protein. We observed a good correlation for high-abundant proteins (upper $50 \%$ of DIA intensities) in all sample types. For low-abundant proteins (lower $50 \%$ of DIA intensities), we only detected a good correlation between DIA and PRM for the LEF. For the more complex samples, however, DIA seemed to underestimate high signal intensities, resulting in poor correlation with the PRM data.

As the main application of both methods is the quantitative comparison of the lysosomal proteome between different states, we performed a spike-in experiment to simulate constitutive upregulation of the whole lysosomal proteome and analyzed the sample by both PRM and DIA, applying 60 min gradients for both approaches. For this purpose, we combined LEF and LTL in a 1 to 5 ratio and compared the data to LTL samples without spike-in (Figure 1). In theory, as LEFs contain higher amounts of lysosomal proteins, this should result in a general increase of intensity for all lysosomal proteins present in the sample.

For both approaches, the number of detected lysosomal proteins increased in comparison to LTL without spike-in, while the increase for PRM was 50\% higher compared to DIA (223 to 243 for DIA and 278 to 308 for PRM, Figure $7 \mathrm{~b}$ ). Subsequently, we investigated the fold change ratios for proteins identified in all samples with both approaches (Figure 7c). We detected a median increase of intensity of 1.8 for PRM and 1.2 for DIA. When investigating values for individual proteins, we observed a discrepancy of $\geq 30 \%$ between fold change values acquired by DIA and PRM for $75 \%$ of proteins (average CV for DIA and PRM analysis of LTLs: 16\% and 7\%). While 142 proteins were detected with a higher value in PRM, only 35 were higher in the DIA data (Figure 7d). Classification of proteins based on their fold change values between the spike-in and the LTL sample further showed that DIA failed to detect any increase in signal intensity for 81 proteins upon spike-in, while this was only the case for 10 proteins in the PRM data (Figure 7e). Subsequently, we investigated if this effect was related to the abundance of individual proteins, as we observed markedly reduced correlation coefficients between DIA and PRM for lower abundant proteins in LTL samples (Figure 7a). Along this line, we grouped all proteins based on their abundance relative to the highest/lowest abundant protein in the respective dataset and plotted the observed fold change ratios for the individual groups (Figure 7f). While we observed highly similar fold change value distributions between LTL and spike-in samples for proteins across the whole range of abundance for the PRM data, a clear shift in the pattern of the DIA data was visible. Relative to the PRM data, DIA reported higher fold change ratios for low-abundant proteins while it resulted in lower 
values for high-abundant proteins. Taken together, these data indicate that PRM is better suited for the quantification of changes in the lysosomal proteome of LTL, which is mainly related to the better performance for the highest- and lowest-abundant lysosomal proteins in the dataset.

(a)

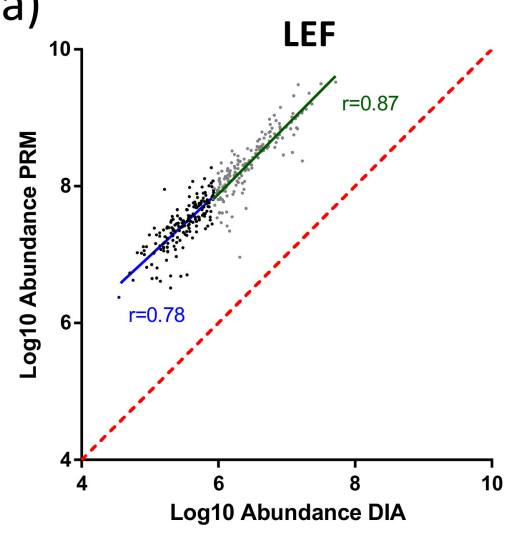

(b)

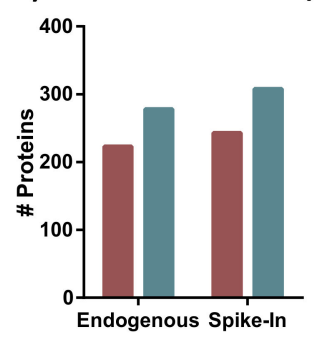

(c)

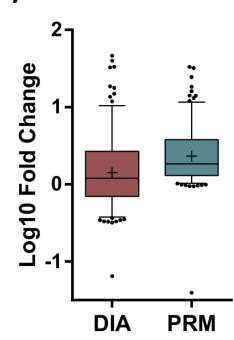

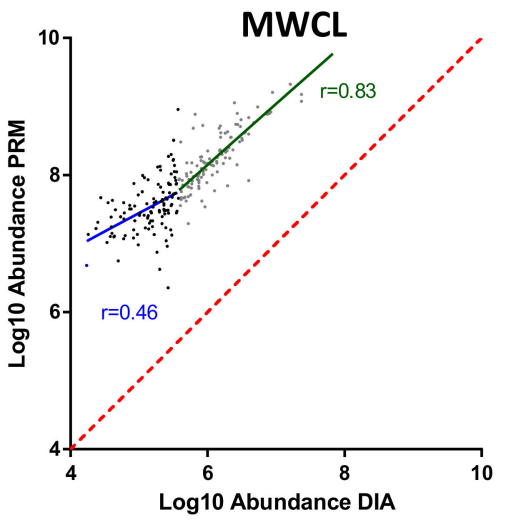

(d)

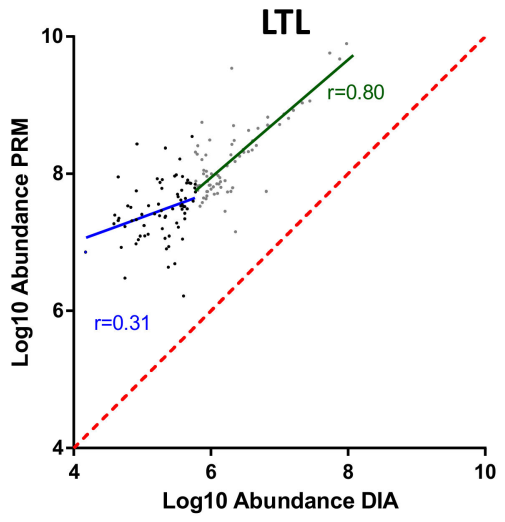

(e)

(f)
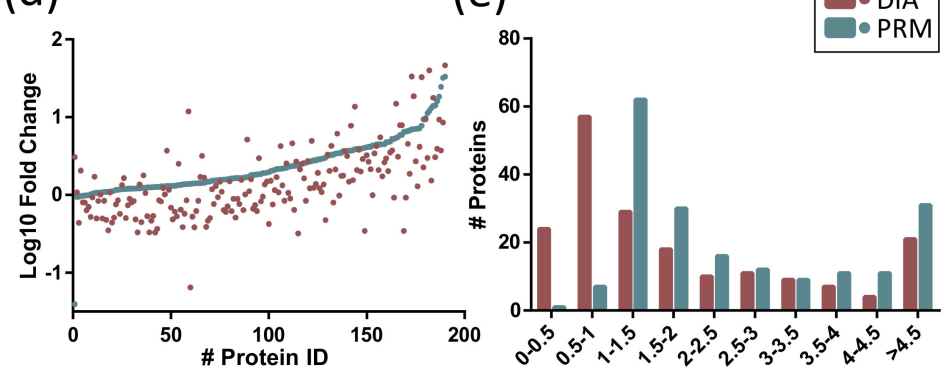

Enrichment Factor

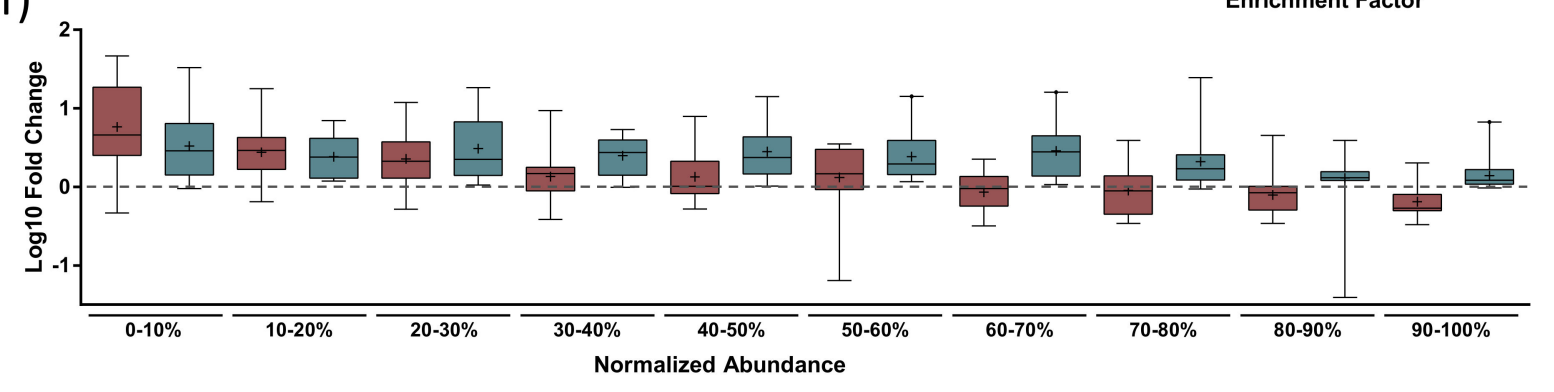

Figure 7. Comparison of DIA and PRM analysis of lysosomal proteins. (a) Correlation of normalized peptide signal intensities for DIA and PRM runs. Proteins are sorted based on their intensity in DIA measurements and grouped into two groups based on their intensity (upper/lower $50 \%$ of proteins). For each population, a linear regression analysis was performed and the respective correlation coefficient $(r)$ is indicated. (b) Identification of proteins in LTL with and without spike-in of LEFs. (c,d) Fold change values for individual lysosomal proteins in LTL with spike-in of LEFs. (e) Frequency of proteins within distinct fold change quantiles for DIA and PRM data for ratios of LTL with/without spike-in of LEFs. (f) Protein fold change values for LTL with/without spike-in of LEFs. Proteins are grouped based on their abundance in the respective dataset relative to the highest/lowest-abundant protein. LEF: lysosome-enriched fractions from mouse embryonic fibroblasts; MWCL: mouse embryonic fibroblast whole cell lysates; LTL: whole liver tissue lysate.

\section{Conclusions}

In the present study, we analyzed the lysosomal proteome in samples of varying complexity by DIA and PRM. While both methods were well-suited for the analysis of lysosomal proteins in all samples, differences between the approaches became apparent that were mostly related to sample complexity. DIA identified more proteins in lower complexity samples and at longer gradients, since it was not limited by a predefined list of 
peptides, as was the case for the PRM analyses. Furthermore, no assay development was necessary for DIA analyses, thus greatly reducing the amount of time needed. For peptides covered by both approaches, DIA and PRM performed similarly for lower complexity LEFs, while PRM outperformed DIA in both MWCL and LTL. Especially for the quantification of protein level changes in LTL, PRM was able to identify significantly higher numbers of protein level alterations than DIA, which reported no change in abundance for a high number of proteins. Therefore, for the analysis of highly complex samples, such as whole tissue lysates, PRM presents the method of choice. Our developed PRM assay allows for the direct analysis of the lysosomal proteome from small amounts of whole tissue samples, without the need for lysosome enrichment, extending the toolbox for the investigation of the lysosomal proteome in complex samples.

Supplementary Materials: The following are available online at https:/ / www.mdpi.com/2227-738 2/9/1/4/s1, Table S1: Reference list of high confidence lysosomal proteins and peptides covered by PRM assay. Supplementary Table S2: DIA Data, direct output from Spectronaut as well as processed data. Supplementary Table S3: PRM data, direct output from Skyline. Supplementary Table S4: PRM data filtered for dot $\mathrm{p}$ values $>0.7$ and further analyses. Supplementary Figure S1: Dot product threshold determination for the acceptance of PRM data. Figure S2: Overlap of identified proteins from PRM and DIA runs.

Author Contributions: Conceptualization, D.W.; methodology, P.M., A.S., and J.S.; validation, P.M., A.S., and J.S.; formal analysis, P.M., A.S., and J.S.; investigation, P.M., A.S., and J.S.; resources, P.M., and A.S.; writing — original draft preparation, A.S., and D.W.; writing-review and editing, P.M., A.S., J.S., and D.W.; visualization, P.M., A.S., and J.S.; supervision, D.W.; project administration, D.W.; funding acquisition, D.W. All authors have read and agreed to the published version of the manuscript.

Funding: This research was funded by the Deutsche Forschungsgemeinschaft, grant number FOR2625, and the Studienstiftung des Deutschen Volkes.

Institutional Review Board Statement: Not applicable.

Informed Consent Statement: Not applicable.

Data Availability Statement: The mass spectrometry proteomics data have been deposited to the ProteomeXchange Consortium via the PRIDE [35] partner repository with the dataset identifier PXD023278.

Acknowledgments: The authors would like to thank Robert Hardt for helpful discussions, Shiva Ahmadi for help with liver samples, and Fatema Akter as well as Srigayatri Ponnaiyan for help with lysosome-enriched fractions.

Conflicts of Interest: The authors declare no conflict of interest.

\section{References}

1. De Duve, C.; Wattiaux, R. Functions of Lysosomes. Annu. Rev. Physiol. 1966, 28, 435-492. [CrossRef] [PubMed]

2. Perera, R.M.; Zoncu, R. The Lysosome as A Regulatory Hub. Annu. Rev. Cell Dev. Biol. 2016, 32, 223-253. [CrossRef] [PubMed]

3. Platt, F.M.; d'Azzo, A.; Davidson, B.L.; Neufeld, E.F.; Tifft, C.J. Lysosomal Storage Diseases. Nat. Rev. Dis. Primers 2018, 4, 27. [CrossRef] [PubMed]

4. Ballabio, A.; Gieselmann, V. Lysosomal Disorders: From Storage to Cellular Damage. Biochim. Biophys. Acta 2009, 1793, 684-696. [CrossRef] [PubMed]

5. Jeyakumar, M.; Dwek, R.A.; Butters, T.D.; Platt, F.M. Storage Solutions: Treating Lysosomal Disorders of The Brain. Nat. Rev. Neurosci. 2005, 6, 713-725. [CrossRef] [PubMed]

6. Perera, R.M.; Stoykova, S.; Nicolay, B.N.; Ross, K.N.; Fitamant, J.; Boukhali, M.; Lengrand, J.; Deshpande, V.; Selig, M.K.; Ferrone, C.R.; et al. Transcriptional Control of Autophagy-Lysosome Function Drives Pancreatic Cancer Metabolism. Nature 2015, 524, 361-365. [CrossRef]

7. DePaolo, J.; Goker-Alpan, O.; Samaddar, T.; Lopez, G.; Sidransky, E. The Association Between Mutations in The Lysosomal Protein Glucocerebrosidase And Parkinsonism. Mov. Disord. 2009, 24, 1571-1578. [CrossRef]

8. Chi, C.; Riching, A.S.; Song, A.K. Lysosomal Abnormalities in Cardiovascular Disease. Int. J. Mol. Sci. 2020, 21, 811. [CrossRef]

9. Ballabio, A.; Bonifacino, J.S. Lysosomes as Dynamic Regulators of Cell and Organismal Homeostasis. Nat. Rev. Mol. Cell Biol. 2019, 2019, 1-18. [CrossRef] 
10. Settembre, C.; Di Malta, C.; Polito, V.A.; Arencibia, M.G.; Vetrini, F.; Erdin, S.; Erdin, S.U.; Huynh, T.; Medina, D.; Colella, P.; et al. Tfeb Links Autophagy to Lysosomal Biogenesis. Science 2011, 332, 1429-1433. [CrossRef]

11. Zhang, C.S.; Jiang, B.; Li, M.; Zhu, M.; Peng, Y.; Zhang, Y.L.; Wu, Y.Q.; Li, T.Y.; Liang, Y.; Lu, Z.; et al. The Lysosomal V-AtpaseRagulator Complex Is A Common Activator for Ampk And Mtorc1, Acting as A Switch Between Catabolism and Anabolism. Cell Metab. 2014, 20, 526-540. [CrossRef] [PubMed]

12. Aebersold, R.; Mann, M. Mass-Spectrometric Exploration of Proteome Structure and Function. Nature 2016, 537, 347-355. [CrossRef] [PubMed]

13. Akter, F.; Ponnaiyan, S.; Koegler-Mohrbacher, B.; Bleibaum, F.; Damme, M.; Renard, B.Y.; Winter, D. Multi Cell Line Analysis of Lysosomal Proteomes Reveals Unique Features and Novel Lysosomal Proteins. bioRxiv 2020. [CrossRef]

14. Singh, J.; Kaade, E.; Muntel, J.; Bruderer, R.; Reiter, L.; Thelen, M.; Winter, D. Systematic Comparison of Strategies for The Enrichment of Lysosomes by Data Independent Acquisition. J. Proteome Res. 2020, 19, 371-381. [CrossRef] [PubMed]

15. Thelen, M.; Winter, D.; Braulke, T.; Gieselmann, V. Silac-based comparative proteomic analysis of lysosomes from mammalian cells using Lc-Ms/Ms. In Lysosomes: Methods and Protocols; Öllinger, K., Appelqvist, H., Eds.; Springer: New York, NY, USA, 2017; pp. 1-18.

16. Wyant, G.A.; Abu-Remaileh, M.; Frenkel, E.M.; Laqtom, N.N.; Dharamdasani, V.; Lewis, C.A.; Chan, S.H.; Heinze, I.; Ori, A.; Sabatini, D.M. Nufip1 Is A Ribosome Receptor for Starvation-Induced Ribophagy. Science 2018, 360, 751-758. [CrossRef] [PubMed]

17. Zoncu, R.; Bar-Peled, L.; Efeyan, A.; Wang, S.; Sancak, Y.; Sabatini, D.M. Mtorc1 Senses Lysosomal Amino Acids Through an Inside-Out Mechanism That Requires the Vacuolar H+-Atpase. Science 2011, 334, 678-683. [CrossRef] [PubMed]

18. Shi, T.; Song, E.; Nie, S.; Rodland, K.D.; Liu, T.; Qian, W.J.; Smith, R.D. Advances in Targeted Proteomics and Applications To Biomedical Research. Proteomics 2016, 16, 2160-2182. [CrossRef]

19. Bilbao, A.; Varesio, E.; Luban, J.; Strambio-De-Castillia, C.; Hopfgartner, G.; Müller, M.; Lisacek, F. Processing Strategies and Software Solutions for Data-Independent Acquisition in Mass Spectrometry. Proteomics 2015, 15, 964-980. [CrossRef]

20. Hoofnagle, A.N.; Becker, J.O.; Oda, M.N.; Cavigiolio, G.; Mayer, P.; Vaisar, T. Multiple-Reaction Monitoring-Mass Spectrometric Assays Can Accurately Measure the Relative Protein Abundance in Complex Mixtures. Clin. Chem. 2012, 58, 777-781. [CrossRef]

21. Lange, V.; Picotti, P.; Domon, B.; Aebersold, R. Selected Reaction Monitoring for Quantitative Proteomics: A Tutorial. Mol. Syst. Biol. 2008, 4, 222. [CrossRef]

22. Schmidt, A.; Gehlenborg, N.; Bodenmiller, B.; Mueller, L.N.; Campbell, D.; Mueller, M.; Aebersold, R.; Domon, B. An Integrated, Directed Mass Spectrometric Approach for In-Depth Characterization of Complex Peptide Mixtures. Mol. Cell. Proteom. 2008, 7, 2138-2150. [CrossRef] [PubMed]

23. Damaghi, M.; Tafreshi, N.K.; Lloyd, M.C.; Sprung, R.; Estrella, V.; Wojtkowiak, J.W.; Morse, D.L.; Koomen, J.M.; Bui, M.M.; Gatenby, R.A.; et al. Chronic Acidosis in The Tumour Microenvironment Selects for Overexpression of Lamp2 in the Plasma Membrane. Nat. Commun. 2015, 6, 8752. [CrossRef] [PubMed]

24. Sjödin, S.; Öhrfelt, A.; Brinkmalm, G.; Zetterberg, H.; Blennow, K.; Brinkmalm, A. Targeting Lamp2 In Human Cerebrospinal Fluid with A Combination of Immunopurification and High Resolution Parallel Reaction Monitoring Mass Spectrometry. Clin. Proteom. 2016, 13, 4. [CrossRef] [PubMed]

25. Ivry, S.L.; Knudsen, G.M.; Caiazza, F.; Sharib, J.M.; Jaradeh, K.; Ravalin, M.; O’Donoghue, A.J.; Kirkwood, K.S.; Craik, C.S. The Lysosomal Aminopeptidase Tripeptidyl Peptidase 1 Displays Increased Activity in Malignant Pancreatic Cysts. Biol. Chem. 2019, 400, 1629-1638. [CrossRef] [PubMed]

26. Ponnaiyan, S.; Akter, F.; Singh, J.; Winter, D. Comprehensive Draft of The Mouse Embryonic Fibroblast Lysosomal Proteome by Mass Spectrometry Based Proteomics. Sci. Data 2020, 7, 1. [CrossRef]

27. Muntel, J.; Gandhi, T.; Verbeke, L.; Bernhardt, O.M.; Treiber, T.; Bruderer, R.; Reiter, L. Surpassing 10000 Identified and Quantified Proteins in A Single Run by Optimizing Current Lc-Ms Instrumentation and Data Analysis Strategy. Mol. Omics 2019, 15, 348-360. [CrossRef]

28. Winter, D.; Steen, H. Optimization of Cell Lysis and Protein Digestion Protocols for The Analysis of Hela S3 Cells by Lc-Ms/Ms. Proteomics 2011, 11, 4726-4730. [CrossRef]

29. Bruderer, R.; Bernhardt, O.M.; Gandhi, T.; Reiter, L. High-Precision Irt Prediction in The Targeted Analysis of Data-Independent Acquisition and Its Impact on Identification and Quantitation. Proteomics 2016, 16, 2246-2256. [CrossRef]

30. Rosenberger, G.; Bludau, I.; Schmitt, U.; Heusel, M.; Hunter, C.L.; Liu, Y.; MacCoss, M.J.; MacLean, B.X.; Nesvizhskii, A.I.; Pedrioli, P.G.; et al. Statistical Control of Peptide and Protein Error Rates in Large-Scale Targeted Data-Independent Acquisition Analyses. Nat. Methods 2017, 14, 921-927. [CrossRef]

31. Pino, L.K.; Searle, B.C.; Bollinger, J.G.; Nunn, B.; MacLean, B.; MacCoss, M.J. The Skyline Ecosystem: Informatics for Quantitative Mass Spectrometry Proteomics. Mass Spectrom. Rev. 2020, 39, 229-244. [CrossRef]

32. Hulsen, T.; de Vlieg, J.; Alkema, W. Biovenn-A Web Application for The Comparison and Visualization of Biological Lists Using Area-Proportional Venn Diagrams. BMC Genom. 2008, 9, 488. [CrossRef] [PubMed]

33. Zubarev, R.A.; Makarov, A. Orbitrap Mass Spectrometry. Anal. Chem. 2013, 85, 5288-5296. [CrossRef] [PubMed] 
34. Frewen, B.E.; Merrihew, G.E.; Wu, C.C.; Noble, W.S.; MacCoss, M.J. Analysis of Peptide Ms/Ms Spectra from Large-Scale Proteomics Experiments Using Spectrum Libraries. Anal. Chem. 2006, 78, 5678-5684. [CrossRef] [PubMed]

35. Perez-Riverol, Y.; Csordas, A.; Bai, J.; Bernal-Llinares, M.; Hewapathirana, S.; Kundu, D.J.; Inuganti, A.; Griss, J.; Mayer, G.; Eisenacher, M.; et al. The Pride Database and Related Tools and Resources In 2019: Improving Support for Quantification Data. Nucleic Acids Res. 2019, 47, D442-D450. [CrossRef] [PubMed] 